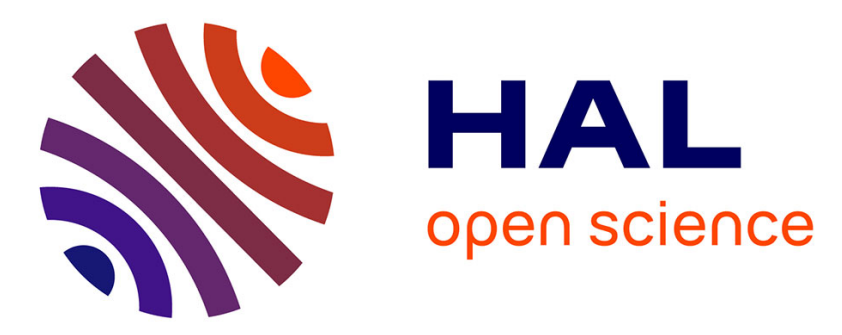

\title{
On the modelling of wavepacket scattering noise with coherence effects
}

Filipe da Silva, Peter Jordan, André Cavalieri

\section{To cite this version:}

Filipe da Silva, Peter Jordan, André Cavalieri. On the modelling of wavepacket scattering noise with coherence effects. Journal of the Acoustical Society of America, 2019, 146 (6), 10.1121/1.5140190 . hal-02380357

\section{HAL Id: hal-02380357 \\ https://hal.science/hal-02380357}

Submitted on 26 Nov 2019

HAL is a multi-disciplinary open access archive for the deposit and dissemination of scientific research documents, whether they are published or not. The documents may come from teaching and research institutions in France or abroad, or from public or private research centers.
L'archive ouverte pluridisciplinaire HAL, est destinée au dépôt et à la diffusion de documents scientifiques de niveau recherche, publiés ou non, émanant des établissements d'enseignement et de recherche français ou étrangers, des laboratoires publics ou privés. 


\title{
On the modelling of wavepacket scattering noise with coherence effects
}

\author{
Filipe D. da Silva, ${ }^{1}$ Peter Jordan, ${ }^{2}$ and André V. G. Cavalieri ${ }^{3}$ \\ ${ }^{1)}$ Departamento de Engenharias da Mobilidade, Universidade Federal de Santa \\ Catarina, Joinville-SC, 89219-600, Brazil \\ ${ }^{2}$ PPRIME Institute, CNRS - ENSMA - Univ. Poitiers, Poitiers, 86036, \\ France \\ ${ }^{3}$ Instituto Tecnológico de Aeronáutica, São José dos Campos, 12228-900, \\ Brazil
}

(Dated: 22 September 2019) 
An investigation of a wavepacket model for free-jet and jet-surface interaction noise was conducted. The source term for the axisymmetric mode was extracted from a Mach 0.9 jet Large Eddy Simulation (LES) and employed to adjust the parameters of a simple source model. Streamwise coherence decay, in particular, was considered. The source model was propagated with both the free-field and tailored Green's function for a semi-infinite flat plate positioned at a distance of $r / D=1$ from the jet axis. First, a model with radial content was considered. Significant deviations were observed in the prediction of the low-angle directivity of the isolated jet as well as in the reproduction of the characteristics of the source field. However, the effects of trailing edge noise were well reproduced. The installed jet case, (Added: at the region dominated by trailing-edge scattering), showed very little sensitivity to the coherence decay, a crucial feature in the isolated jet case. (Deleted: Through the use of a line source, involving greater ease in the adjustments, the models were capable to predict the acoustic fields in all studied configurations in low Strouhal number.) The modelling of installed jets proved to be much simpler, since the results were much less sensitive to the characteristics of the source.

a)filipe.dutra@ufsc.br; Corresponding author. 


\section{INTRODUCTION}

The adverse effects arising from the integration of engines with the aircraft structure have gained importance with the rise of stricter noise regulations. Among the sources of noise due to this integration are the interaction of the jet flow with wings and flaps. In this case the problem is intensified by the close coupling between the engine and the wing, due to the use of high bypass ratio engines.

The effects caused by the presence of solid surfaces near turbulent regions have been known for decades. Curle ${ }^{1}$ presented an extension of Lighthill's theory ${ }^{2}$ in order to account for the influence of solid boundaries on the acoustic field. In addition to the jet quadrupoles, surface pressure leads to the appearance of dipole sources, which have stronger far-field contribution at low Mach numbers. Furthermore, Ffowcs Williams and $\mathrm{Hall}^{3}$ studied the case involving semi-infinite plates using a tailored Green's function. In this case, the deduced edge scattering source is even more efficient than both the free turbulence in Lighthill's theory or compact surfaces in Curle's work.

Wavepackets are intermittent, advecting disturbances that are correlated over distances far exceeding the integral scales of turbulence ${ }^{4}$. Several experimental and theoretical studies highlighted the importance of such structures in jet noise ${ }^{5-8}$. Theoretical models based on wavepackets have been shown to capture many of the main characteristics of the far-field noise for isolated ${ }^{8,9}$ and installed jet $\operatorname{cases}^{10-12}$. However, inconsistencies are present as the absolute noise levels are under predicted by linear dynamic models, such as the Parabolised Stability Equations. These linear models have unit coherence in contrast with turbulent 
flows, in which coherence decays with distance. This issue was investigated by Cavalieri and Agarwal $^{13}$ who show the match of the coherence of the original source to be fundamental for correctly predicting the sound field. Studies showed that the coherence decay found in turbulent jets, when included in the linear models, raise the radiating efficiency of the source $^{13-15}$.

Regarding installation effects, Cavalieri et al. ${ }^{10}$ modelled a jet-plate interaction case using a wavepacket source model along with a tailored Green's function and a boundary element method. The scattering of the non-compact wavepackets in the jet near field was found to be responsible for the amplification of the noise due to the presence of the plate. The used model was identified from far-field measurements of free jet noise. Further studies are needed to evaluate the pertinence of the model, and it would be important to use data from jet turbulence to construct a source. Moreover the role of jitter/coherence decay is apparently weaker in installed jet noise according to Nogueira et al. ${ }^{12}$, but the cited work dealt with a model problem. Again, the use of turbulence data may help addressing this question in a more definite way. (Added: The use of model sources and Lighthill's equation for predicting installed jet noise has been also explored by Refs. 16, 17 and 18.)

The objective of this paper is to analyse wavepacket source models, obtained from numerical flow data, for the prediction of free-jet and jet-plate interaction noise. We first investigate simplifications on the Lighthill's source term and their relevance to the total sound field using the LES data from Ref. 19. Subsequently, the same numerical data is used to obtain parameters of kinematic wavepacket source models, and the produced noise field 
was obtained using both the free-field Green's function and a tailored Green's function for a semi-infinite flat plate.

This work is organized as follows. In section II, we describe the used geometries and the main equations. In section III, we present the acoustic results from a simplified source term extracted from the LES data. Results for the model source are presented in section IV. Finally, section V presents the main conclusions.

\section{METHODS}

\section{A. Geometry}

The study presented herein is based on the experiments conducted at PPRIME Institute, Poitiers, France ${ }^{11}$. The experiment consists of a nozzle of diameter $D=50 \mathrm{~mm}$ with a flat plate positioned at its vicinity, as shown in the sketch of Fig. 1. (Added: The plate has a chord of $9 D$ and a span length of $15 D$.) A total of 324 acoustic field measurements were taken on a cylindrical surface of radius $R=14.2 D$. The studied configuration had a jet acoustic Mach number $M_{a}=0.9$ and Reynolds number $R e_{j}=\rho_{j} U_{j} D / \mu_{j} \approx 1 \times 10^{6}$. The trailing-edge position at the center of the plate is $x / D=4$ and $r / D=1$ is the position relative to the nozzle axis; here we consider a cylindrical coordinate system, with origin at the nozzle exit, given by $(x, r, \phi)$, corresponding to streamwise, radial and azimuthal coordinates, respectively. 


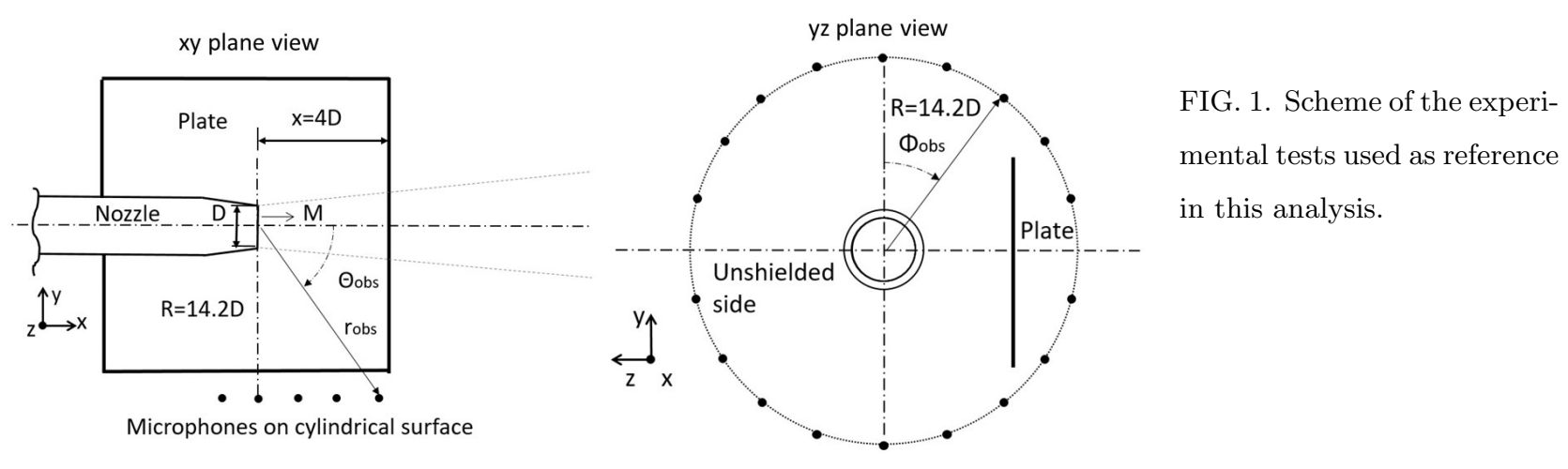

\section{B. Large eddy simulation database}

The large eddy simulation (LES) database used corresponds to the same jet conditions and nozzle geometry, considering only the free-jet case. (Added: The jet is isothermal in the simulation.) The simulation was performed using the compressible flow solver "Charles" developed at Cascade Technologies, and reproduces the configuration from the companion experiment conducted at the PPRIME Institute, Poitiers, France.

The LES methodology, grid resolution study and validation with experiments are presented in details in Brès et al. ${ }^{19}$. The instantaneous values of the primitives variables were interpolated from the original unstructured LES grid onto structured cylindrical grids in the jet plume and in the nozzle pipe. These cylindrical grids were designed such that the resolution approximately corresponds to the underlying LES resolution. For the jet plume, the cylindrical grid extends to $0 \leq x / D \leq 30,0 \leq r / D \leq 6$, with $\left(n_{x}, n_{r}, n_{\phi}\right)=(626,138,128)$, where $n_{x}, n_{r}$ and $n_{\phi}$ are the number of points in the streamwise, radial and azimuthal direction, respectively. The points are equally-spaced in the azimuthal direction to enable simple azimuthal decomposition in Fourier space. 


\section{Eduction of the source term from LES}

The source term is based on the Lighthill ${ }^{2}$ equation given by,

$$
\frac{\partial^{2} \rho}{\partial t^{2}}-c^{2} \nabla^{2} \rho=\frac{\partial^{2} T_{i j}}{\partial x_{i} \partial x_{j}}
$$

where the Lighthill's tensor, with viscous terms neglected, is defined as, $T_{i j}=\rho U_{i} U_{j}+(p-$ $\left.c^{2} \rho\right) \delta_{i j}$, where $U_{i}$ is the velocity vector, $p$ the pressure, $\rho$ the fluid density, $c$ the speed of sound and $\delta_{i j}$ the Kronecker delta.

We now apply several simplifications to the source term, which nonetheless are expected to retain the main source features for sound generation ${ }^{8,14}$. The entropy term is not considered for an isothermal jet and source density variations are substituted by the mean $\rho_{0}$. Under the hypothesis of the predominance of the momentum term $T_{x x}$, separating mean and fluctuating parts of the streamwise velocity $U_{x}=\overline{U_{x}}+u_{x}$ and taking only the linear part, the source term in cylindrical coordinates is given by:

$$
S^{t}=\frac{\partial^{2} T_{x x}}{\partial x^{2}}=\rho_{0} \frac{\partial^{2}}{\partial x^{2}} 2 \overline{U_{x}} u_{x}(x, r, \phi, t) .
$$

The source is decomposed into azimuthal Fourier modes, as we are interest in just the axissymmetric component:

$$
S^{t}(x, r, m, t)=\frac{1}{2 \pi} \int_{-\pi}^{\pi} S^{t}(x, r, \phi, t) e^{i m \phi} d \phi
$$


and only $m=0$ is considered,

$$
S^{t}=\frac{\partial^{2} T_{x x}}{\partial x^{2}}=\rho_{0} \frac{\partial^{2}}{\partial x^{2}} 2 \overline{U_{x}} u_{x}(x, r, m=0, t)
$$

(Added: By neglecting $T_{r r}$ and $T_{x r}$ radiation is expected to be predominant towards low angles $^{22}$. Also, non-linear terms should be significant only at sideline angles as studied by Freund ${ }^{?}$. Furthermore, mode 0 is expected to be dominant at at polar angles close to $30^{\circ}$, while the contributions of modes 1 and 2 are more significant at high angles ${ }^{8,24}$. )

The superscript $t$ implies the quantities in the time domain. This source term is Fourier transformed with using the following convention

$$
S=\int_{-\infty}^{\infty} S^{t} e^{-i \omega t} d t
$$

The source distribution is obtained from the LES database, which is for a free jet. It will be used in calculations of free and installed jet noise, as will be presented in the next sections. For the installed jet calculations, there is an implicit assumption that the presence of a neighbouring plate does not modify substantially the source field. The accuracy of the calculation may serve as an a posteriori check of this assumption.

\section{Computation of the acoustic pressure at observer positions}

The source distribution obtained from the LES database, with the simplifications described in the previous section, allow an estimation of the radiated sound in free- and installed-jet configurations. The calculation is done in the frequency domain, based on the integral solution of the inhomogeneous Helmholtz equation, resulting from the Fourier 
transform of Lighthill's equation. As we are dealing with turbulent properties, and so non square-integrable functions, it is more appropriate to work with two-point statistics. In this sense, the Power Spectral Density (PSD) of the sound pressure field can be obtained from the Cross Spectral Density (CSD) of the source field by:

$$
\begin{aligned}
& \left\langle p(\mathbf{x}, m, \omega) p^{*}(\mathbf{x}, m, \omega)\right\rangle= \\
& \int_{V} \int_{V}\left\langle S(\mathbf{y}, m, \omega) S^{*}(\mathbf{z}, m, \omega)\right\rangle G(\mathbf{x}, \mathbf{y}, \omega) G^{*}(\mathbf{x}, \mathbf{z}, \omega) d \mathbf{y} d \mathbf{z}
\end{aligned}
$$

For the free jet case, $G=G_{0}$ is the free-field Green's function given as,

$$
G_{0}=\frac{e^{-i k R}}{4 \pi R}
$$

where $R$ is the distance between source an observer and $k=\omega / c_{0}$ the acoustic wavenumber.

For the installed jet case, the problem is modelled as the jet scattering from a semiinfinite plate. This approach has been used with wavepacket models and was shown to be representative of the problem, although some deviations occur due to the differences to the real case (finite plate) ${ }^{10-12}$. The tailored Green's function from Ffowcs Williams and Hall ${ }^{3}$ is used,

$$
4 \pi G_{t}=\frac{e^{\frac{1}{4} i \pi}}{\sqrt{\pi}}\left\{\frac{e^{-i R}}{R} \int_{-\infty}^{u_{R}} e^{-i u^{2}} d u+\frac{e^{-i R^{\prime}}}{R^{\prime}} \int_{-\infty}^{u_{R^{\prime}}} e^{-i u^{2}} d u\right\}
$$

where,

$$
U_{R}=2\left(\frac{k r r_{0}}{D+R}\right)^{\frac{1}{2}} \cos \frac{\theta-\theta_{0}}{2}
$$




$$
\begin{aligned}
& u_{R^{\prime}}=2\left(\frac{k r r_{0}}{D+R^{\prime}}\right)^{\frac{1}{2}} \cos \frac{\theta+\theta_{0}}{2}, \\
& D=\left\{\left(r-r_{0}\right)^{2}+\left(z-z_{0}\right)^{2}\right\}^{\frac{1}{2}} .
\end{aligned}
$$

The tailored Green's function on its original form, implies a cylindrical coordinate system with axial coordinate aligned with the trailing edge. The position of the source point in this coordinate system is $\left(r_{0}, \theta_{0}, z_{0}\right)$ and the observer $(r, \theta, z) . R$ is then the distance between source and observer and $R^{\prime}$ is the distance between the source's image and observer.

As numerical derivatives of the turbulent data generated undesirable noise in the results, the derivatives were transferred to the Green's function. The derivatives were performed analytically and the derivation from Nogueira et $a l .{ }^{12}$ is used for the tailored Green's function.

The whole available LES time data was used, with a total non-dimensional time of $t c_{0} / D=2000$ and a sampling rate of $\Delta t c_{0} / D=0.2$. (Added: The source domain extended $0 \leq x / D \leq 30,0 \leq r / D \leq 3$, with $\left(n_{x}, n_{r}\right)=(328,58)$ points. $)$ The Cross Spectral Densities (CSDs) were calculated using Welch's method ${ }^{20}$ using block size of Nfft $=256$ timesteps, overlap of $75 \%$. Sensitivity tests for the window function were conducted. Acoustics results obtained from the CSD calculated with rectangular window presented an undesirable dependence with the block size. Nevertheless, results with Hanning window showed much lower sensitivity to the block size. Based on these results, the Hanning window was chosen for further analyses. 


\section{E. Model sources}

Model sources used in this work are inspired by the wavepacket line source model presented by Crow $^{21}\left(\right.$ see Crighton ${ }^{22}$ ). This source and variations were used in Refs. 8, 10-12, some of them introducing radial information with the insertion of radial profiles obtained via PSE solutions. Based on the coherence modelling proposed by Cavalieri and Agarwal ${ }^{13}$ the following source model is employed:

$$
\begin{aligned}
& \left\langle S\left(\mathbf{x}_{\mathbf{1}}, \omega\right) S^{*}\left(\mathbf{x}_{\mathbf{2}}, \omega\right)\right\rangle=A \exp \left(-\frac{\left(x_{1}-X_{c}\right)^{2}}{L_{x}^{2}}-\frac{\left(x_{2}-X_{c}\right)^{2}}{L_{x}^{2}}\right) \\
& \operatorname{erfc}\left(\frac{\alpha\left(x_{1}-X_{c}\right)}{\sqrt{2} L_{x}}\right) \operatorname{erfc}\left(\frac{\alpha\left(x_{2}-X_{c}\right)}{\sqrt{2} L_{x}}\right) \exp \left(-\frac{\left(r_{1}-R_{c}\right)^{2}}{L_{r}^{2}}-\frac{\left(r_{2}-R_{c}\right)^{2}}{L_{r}^{2}}\right) \\
& \exp \left[-i k_{h}\left(x_{1}-x_{2}\right)\right] \exp \left(-\frac{\left|\mathbf{x}_{\mathbf{1}}-\mathbf{x}_{\mathbf{2}}\right|^{2}}{L_{c}^{2}}\right),
\end{aligned}
$$

such that the $C S D$ of the source is represented by 7 global parameters. $A$ represents the maximum $P S D$ amplitude, whose streamwise decay is represented by an asymmetric Gaussian profile centered at $X_{c}$ with characteristic length $L_{x}$ and $\alpha$ as the skewness parameter. The radial profile is represented by a symmetric Gaussian profile with envelope length $L_{r}$. Centering this second Gaussian at $R_{c}=0.25$ was taken as a reasonable assumption. Streamwise phase difference is defined by the hydrodynamic wavenumber $k_{h}$, related to the phase speed $U_{c} / U_{J}=2 \pi S t / k_{h}$.

Finally the coherence decay is represented by Gaussian profiles of characteristic length

$L_{c}$. The parameters were obtained by minimizing the sum of the squares of the absolute error between the numerical source and the proposed model. 
(Deleted: In order to further simplify the model, a line source approach, similar to Ref.

13 is derived. For a free-jet case, at low St number, with observer at the far-field with $\left(r_{o b s} \gg r_{s}\right)$ it is acceptable to assume radial compactness, leading to an independence of the Green's function with the radial distance. In this way parting from the eq. 6 becomes,

(Deleted: with the CSD of the line source defined as)

(Deleted: This line source can be parametrised in a similar manner to the volumetrie source in eq. 12, leading to)

\section{SOUND RADIATION FROM LES SOURCE TERMS}

\section{A. Isolated jet}

The linearised, $m=0, T_{x x}$ source term extracted from the LES was first propagated by the integration with the free-field Green's function (Eq. 6). As already known ${ }^{8,23,24}$, the axisymmetric mode is dominant at low polar angles, while higher order modes are important at high angles. Only $m=0$ experimental data is show for the free-jet case, while the complete set of azimuthal modes will be used for the installed case.

Results obtained by the integration with the free-field Green's function are shown in Fig. 2 (LES source) for $S t=0.2,0.3$ and 0.75 . The results show good agreement to experimental data for $m=0$ at low angles and $S t$ numbers, reproducing the axisymmetric super directive features $^{8}$. Although the agreement is indeed very good at $S t=0.2$ (Fig. 2(a)), levels are overpredicted at $20^{\circ}$ and $30^{\circ}$ for the other $\mathrm{St}$. 


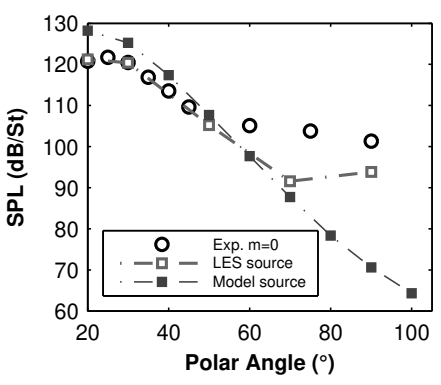

(a) $S t=0.2$

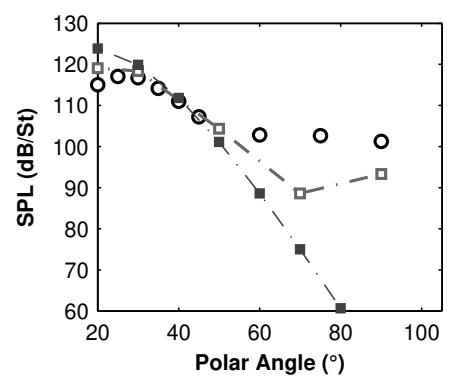

(b) $S t=0.3$

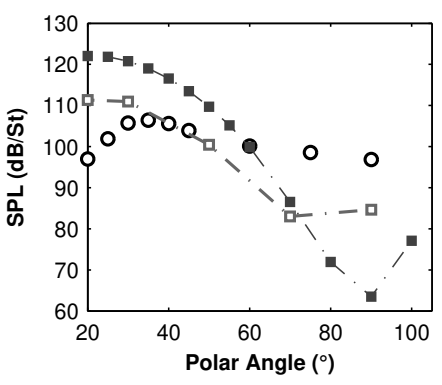

(c) $S t=0.75$

FIG. 2. Comparison of free jet results for the three considered $S t$ numbers.

Neglecting the entropy term in the definition of the source term could be an important issue for these observed deviations, since Bodony and Lele ${ }^{25}$ identified a cancellation effect between the entropy and momentum terms for $S t$ below 0.3 at this radiation direction. However, we should keep in mind that the analysed jet is isothermal, unlike the one considered in the cited study. Here, the disparities become larger with growing $S t$, and which is also aligned with the growing importance of refraction effects. These deviations are expected to reduce once the complete Lighthill's tensor is taken into account.

By looking at angles higher than $60^{\circ}$, we see that levels were underpredicted by about $10 \mathrm{~dB}$. At these directions both $T_{r r}$ and $T_{x r}$ and possibly non-linear terms have considerable contribution to total noise levels, but were not considered in this study ${ }^{22}$. As already expected, more terms and more azimuthal Fourier modes should be accounted for when trying to predict noise at high angles for an isolated jet. 


\section{B. Installed Jet, plate at $r / D=1$}

Fig. 3(a) and 4(a) depict the results for the installed case (Added: (LES source)) for azimuthal angle $\phi_{o b s}=260^{\circ}$, at the unshielded side of the plate (See Fig. 1). For the region dominated by the edge scattering (polar angles around $90^{\circ}$ ), levels were close to the experimental total noise (all modes). This now points out to the dominance of the mode $m=0$ and $T_{x x}$ for installed jet noise, which is in line with the results of Refs. 10 and 12 . This observation supports the study in the following section, where a simplified axisymmetric $T_{x x}$ wavepacket model source is adjusted to predict the installation noise field. (Added: In order to evidence the contribution of the plate to the total noise, the scattered only noise field was included in the same plots.)

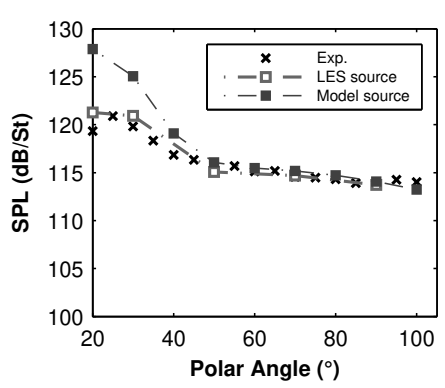

(a) $\phi_{\text {obs }}=260^{\circ}$

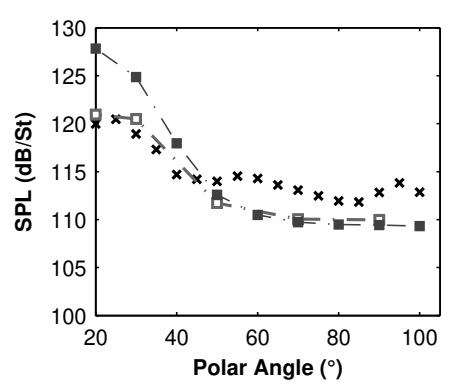

(b) $\phi_{\text {obs }}=220^{\circ}$

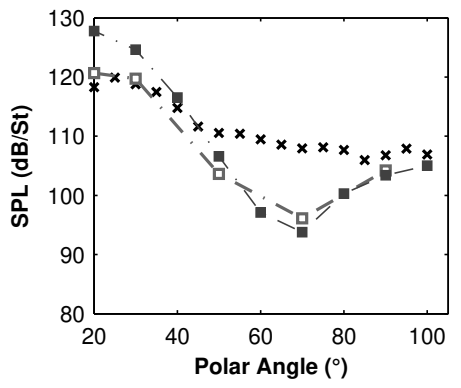

(c) $\phi_{\text {obs }}=180^{\circ}$

FIG. 3. Comparison of installed jet results for $S t=0.2$.

For $S t=0.2$ and 0.3 in Fig. 3(Added: b) and 4(Added: b) at $\phi_{o b s}=220^{\circ}$, results are underpredicted by about 2 to $4 \mathrm{~dB}$. We should bear in mind that the azimuthal directivity of (Replaced: installed-jet replaced with: edge-scattering) noise is of dipole shape, peaking at $\phi=90^{\circ}$ and $270^{\circ}$ and with minima at $\phi=0,180^{\circ 26}$; thus, from $\phi=260^{\circ}$ to $220^{\circ}$ we are moving to a region of lower installation effects. Furthermore, at $M=0.9$, (Replaced: 


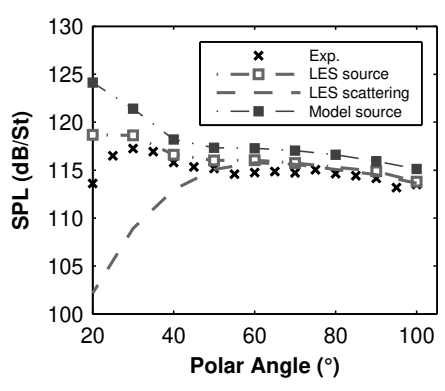

(a) $\phi_{\text {obs }}=260^{\circ}$

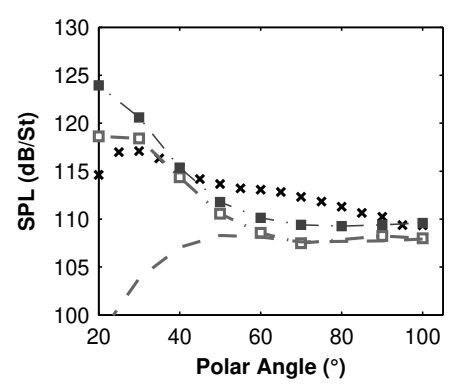

(b) $\phi_{\text {obs }}=220^{\circ}$

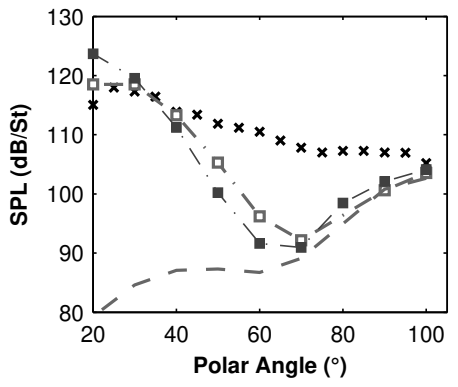

(c) $\phi_{\text {obs }}=180^{\circ}$

FIG. 4. Comparison of installed jet results for $S t=0.3$.

installation replaced with: trailing-edge) effects are not so prominent as in lower Mach number ${ }^{11,27}$. In this sense, in such radiation direction, the free jet could present noise levels comparable to the scattered field. Thus, the remaining terms and modes in the Lighthill source have some contribution at this position. In addition, the differences between the real finite plate and its representation by the semi-infinite plate may also play a role for the observed deviations. This is supported by the presence of level oscillations with the polar angle in the experimental data, potentially caused by the secondary scattering by lateral $\operatorname{edges}^{10}$.

Minor scattering effects are expected in directions parallel to the trailing edge in the case of the semi-infinite plate. As consequence the results for $\phi_{\text {obs }}=180^{\circ}$ (Fig. $3(\mathrm{c})$ ), polar directivity trends are more similar to those observed for the free jet and so, the missing modes and momentum terms are also important. 


\section{SOUND RADIATION FROM MODEL WAVEPACKET SOURCE}

We now benefit from the presented LES data to adjust a simple kinematic wavepacket source model, such as the ones proposed $b^{8,10,13,21}$. One of the objectives here is to explore the effects of the coherence decay in the isolated and installed jet configurations. The resulting model contributes both to the understanding of the underlying physics of the problem, highlighting the relevant wavepacket parameters to predict installation effects.

\section{A. Two-dimensional source model}

The model parameters were obtained by minimising the sum, (Added: over the discretized domain,) of the squares of the absolute error between the LES source (Added: CSD) and the proposed model (Eq. 12) (Added: $\left.\left(\sum^{N} \sum^{N}\left(\left\|C S D_{L E S}-C S D_{\text {model }}\right\|^{2}\right)\right)\right)$. The domain for the fitting was limited between $x=0.5 D$ and $x=15 D$ and $r=0.2 D$ and $r=1 D$ (Added: with a total number of $N$ points). (Added: The process is carried out with the Nelder-Mead Simplex Algorithm ${ }^{28}$ ).The parameters found for each St are summarized in table I. The total relative error $\left(\Delta=\sum^{N} \sum^{N}\left\|C S D_{L E S}-C S D_{\text {model }}\right\| / \sum^{N} \sum^{N}\left\|C S D_{L E S}\right\|\right)$ is shown in the same table. For the noise computation, the model source was defined on a grid extending from $x=-7 L_{x}$ to $x=7 L_{x}$ and from $r=0$ to $r=1 D$, with 25 points in the radial and 256 points in the axial directions. Results were verified to be grid independent with these values. Acoustic results are show in Fig. 2(a) $(S t=0.2)$, Fig. 2(b) $(S t=0.3)$ and Fig. 2(c) $(S t=0.75)$ for the free jet. 
Levels were overpredicted by about $7 \mathrm{~dB}$ for $S t=0.2$ and $4 \mathrm{~dB}$ for $S t=0.3$ at $20^{\circ}$. For all considered $S t$, levels were highly underpredicted over $60^{\circ}$. The low-angle directivity for the axisymmetric mode could only be reasonably captured for $S t=0.2$. Results for $S t=0.75$ reasonably reproduced the directivity trends, however, noise levels were overpredicted by about $10 \mathrm{~dB}$.

Some deviations from the expected low-angle directivity occurred and seem to increase with St. One possible cause are fitting errors. The failure of the model on capturing the sideline noise may not be unsettling, since the axisymmetric mode and even the $T_{x x}$ term do not have the main contributions to the total noise. However, at low angles, the difficulties in predicting both the magnitude and directivity of the sound field do represent an important issue.

With the plate at $r / D=1$, at polar angles dominated by the edge scattering noise and $\phi_{\text {obs }}=260^{\circ}$ (Fig. 3 and 4 ), levels were much closer to the reference experimental and LES data. Deviations were less than $1 \mathrm{~dB}$ for $S t=0.2$ and about $2.5 \mathrm{~dB}$ for $S t=0.3$. Trailingedge effects are not expected to be important at $S t=0.75$ so installed results were omitted for this case.

In Fig. 5, we can see the comparison between experimental and model results for all the measured directivity range, with the plate at $r / D=1, S t=0.3$. The directivity trends could be well captured for azimuthal angles close to the plane perpendicular to the plate, say between $\phi_{o b s}=50^{\circ}$ and $140^{\circ}$ and between $\phi_{o b s}=220^{\circ}$ and $320^{\circ}$. With the results up to this point, we can note that although the wavepacket model showed some deviations in the prediction of free jet noise, specially at high angles and high $S t$, a close match is obtained 
wherever edge scattering is dominant. These results are aligned with the observations from section III and with Cavalieri et al. ${ }^{10}$.
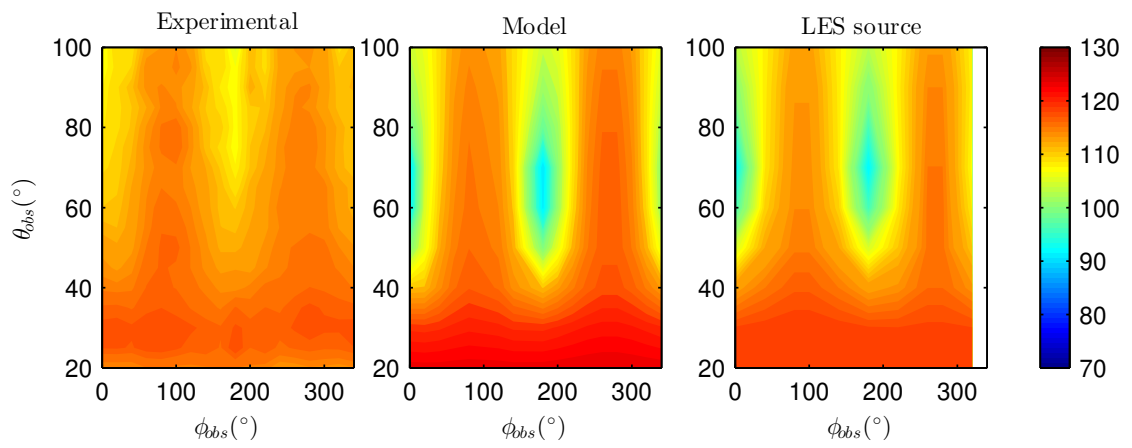

FIG. 5. (Color online) Model source SPL results compared to the experimental data with tailored Green's function at all the measured positions $(S t=0.3)$.

We can note that the relative errors shown in table I are high, specially for $S t=0.75$, and imply a limitation of the used fit. In order to help understanding the possible causes of the found deviations, we now look at the plots of the source region. A comparison between the LES and model sources can be observed in Fig. 6 for the real part of the CSD, for each of the analysed $S t$ and for two fixed radial reference positions at $x_{r e f}=X_{\max }$, where $X_{\max }$ is the streamwise position of the peak power spectral density. The fitting technique provided a good representation of the maximum amplitude at $r=R_{c}$ and source extension around the maximum. Nevertheless, there is a clear mismatch between the radial profiles up to $x / D=5$ and in the jet shear-layer. The use of simple Gaussian profiles for the radial amplitudes and coherence contributed to these deviations, which become larger at higher St.

As depicted Fig. 6, at $S t=0.2$ and reference position $r_{r e f}=0.3$, the model reproduces the main characteristics of the source up between $x / D=5$ and 10. Greater divergence can 

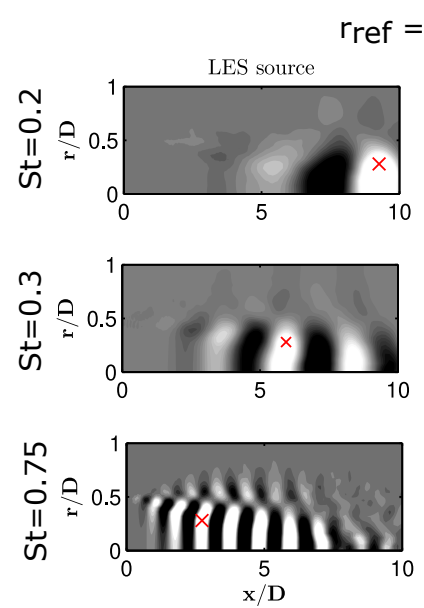

be observed up to $x / D=5$ around the lipline. When the reference point is at the lipline, the disparities are even more evident. Similar conclusions can be drawn for the other $S t$, with even greater disparities at $r_{r e f}=0.5 D$. Deviations from the reference data seem to increase with St, as well as the sound-field results.
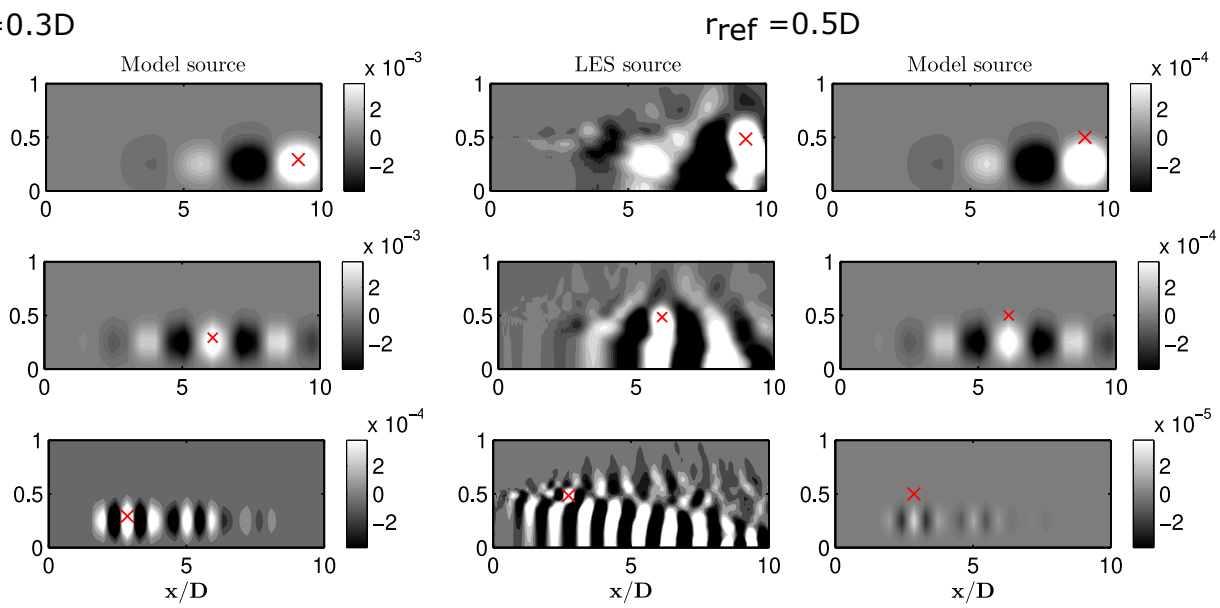

FIG. 6. Comparison between plots of real part of the CSD obtained from the LES data and from the model source with $x_{r e f}=X_{\max }$ at $S t=0.2$ (top), $S t=0.3$ (middle) and $S t=0.75$ (bottom).
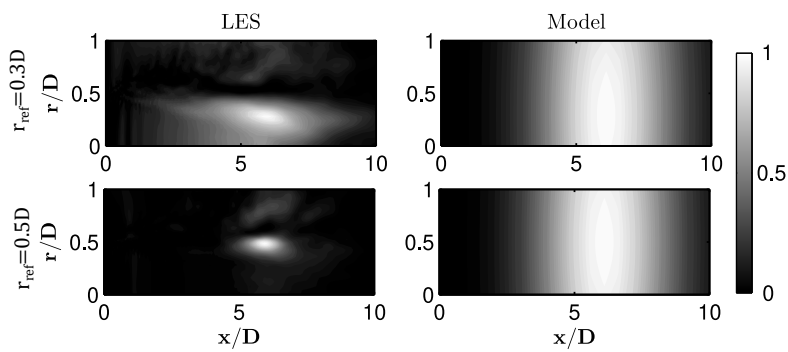

FIG. 7. Comparison between coherence plots obtained from the LES data and from the model source with $x_{r e f}=X_{\max }$ and $S t=0.3$ and two radial reference positions $r_{r e f}=0.3 D$ (top) and $r_{r e f}=0.5 D$ (bottom). 
The comparison of coherence plots is depicted in Fig. 7 for reference points at $r / D=0.3$ and 0.5. The radial coherence decay is obviously not well reproduced by the model. At $r_{r e f}=0.3 D$, the streamwise coherence could be reasonably represented. However, at $r_{r e f}=$ $0.5 D$, a much shorter coherence length is required. This range of coherence length scales in different regions of the source field is not easy to represent with a simple kinematic model.

The content of the Green's functions in the wavenumber space are different ${ }^{12,13}$, which would permit source wavenumbers that are evanescent in the free-field case to generate farfield noise when the jet is close to a flat plate. Slight changes in wave-packet envelope or coherence decay may influence free-jet noise substantially ${ }^{13}$, and lead to little changes for installed jets ${ }^{12}$. That is possibly why the results with the plate were in good agreement with the reference data, despite of the problems observed in the free-jet case.

Although it is not straightforward to tell which are the causes of errors in the acoustic prediction by simply looking at the source field, it is evident that some considerable deviations are present between the fitted wavepacket model and the LES field. These were possibly caused by the use of simple functions for both the amplitudes and coherence, as well as by the neglect of the radial phase differences and radial coherence. However, we are working in the framework of simple kinematic models, and so a parametrisation that would be too complex is not the intention of this work. Furthermore, is important to emphasize that, even with the mentioned simplifications, the model could correctly represent the expected noise characteristics the phenomenon (Added: of trailing-edge scattering, one of the main phenomena of installed jets) (Deleted: for the installed case). Using the same 7-parameter 
model, the next section aims at characterising the sensitivity of the results to the coherence length.

\section{B. Sensitivity to the coherence length}

As already mentioned, the coherence length is an imperative parameter in wavepacket models for isolated jet prediciton ${ }^{13,15}$. We now assess the effects of the variation of this parameter based on the range of values identified in the LES data at $x=X_{c}(S t)$ and $S t=0.3$. Three values were defined: the value at the lipline $\left(L_{c}^{C}=0.94\right)$, the radial average of $L_{c}$ until $r=0.5 D\left(L_{c}^{B}=2.66\right)$ and the fitted value from the previous section $\left(L_{c}^{A}=3\right)$. The unit coherence source, with $L_{c}^{0} \rightarrow \infty$, was also considered.

By looking at the resulting free-jet far-field noise for $S t=0.3$ in Fig. 8, we can see a clear change in the noise directivity with the different $L_{c}$ values. The source with the lipline $L_{c}$ produces very high noise levels in comparison with the LES source. Although reasonable level agreement at low angles was only achieved when considering the larger, original $L_{c}$, only results with $L_{c}^{C}$ reproduced the low-angle directivity trends. As expected ${ }^{13,15}$, the $L_{c}^{0}$ source resulted in levels at least $12 \mathrm{~dB}$ lower than the reference data.

(Added: Fig. 8 also show the results for the total noise from the jet-plate configuration.) The significant changes in level and directivity with $L_{c}$, found for the free jet, does not occur in the installed case at $\phi_{o b s}=260^{\circ}$, over $\theta_{o b s}=50^{\circ}$. In this case, the results are much less dependent on the coherence parameter, and agreement at the edge-scattering-dominated region is good even for the unit coherence source. (Added: The total noise for $L_{c}^{C}$ for the same observer positions differs from the others by about $34 \mathrm{~dB}$ at $\theta_{\text {obs }}=100^{\circ}$, however we 
should not that these levels are very similar to those of the free-jet with the same $L_{c}$ value. However, we cannot observe such differences when looking at the separated scattering field, shown in the same figure.) By looking at (Added: the total noise at) $\phi_{\text {obs }}=220^{\circ}$, we can note that this sensitivity becomes higher as the observer moves to a direction parallel to the plate trailing-edge, where the scattering effects are reduced. (Added: The plots for the scattering-only noise in Fig. 8 confirms that the sensitivity is higher at $\phi_{o b s}=220^{\circ}$.) Indeed, the sensitivity to coherence seems to be much lower wherever edge scattering effects are dominant. Results for $S t=0.2$ showed similar trends to $S t=0.3$ and were omitted here for conciseness.

(Deleted: One extra featured to be observed is that the installed-jet levels are very similar to the free jet for the lowest $L_{c}^{C}$ at high angles, say above $80^{\circ}$. The possible reasons are that the resulting free jet noise is so high, that it masks the seattering noise and that the source is approaching the compact limit, such that scattering effects are reduced, as observed by Nogueira et al. ${ }^{12}$. The main conclusion from these results is that the scattered field demonstrates low sensitivity to the coherence decay, and seems to depend more strongly en other model parameters.)

(Added: When moving the plate away from the jet, say $r / D=1.5$, reduction of the scattering effects is expected. Fig. 8 depicts the results with the plate at at this position. Again, the sensitivity to coherence seems to be very low when edge scattering effects are dominant. As already commented the levels obtained for $L_{c}^{C}$ are influenced by the very high free jet noise.) 
(Added: Finally, the plate was kept at $r / D=1$ and its trailing-edge was moved to other three axial positions $\left(x_{T E}=2 D, x_{T E}=6 D\right.$ and $\left.x_{T E}=8 D\right)$. Results are shown in Fig. 10 for $\phi_{o b s}=260^{\circ}$. As experimental results are not available at these plate positions, only the LES source results are used as reference. For $x_{T E}=2 D$, the noise levels show low sensitivity to the coherence parameter at angles in which edge-scattering is dominant. However, when the plate is positioned very close to the peak source $\left(x_{T E}=6 D\right)$ and further downstream of the peak source $\left(x_{T E}=8 D\right)$, noise results becomes more sensitive to the variation of $L_{c}$. Results from the unit coherence source, for instance, were about $6 \mathrm{~dB} / S t$ lower in comparison with the sources with finite $L_{c}$ values for $\left(x_{T E}=8 D\right)$. This is an interesting finding that suggest that the results become more sensitive to the coherence parameter as the trailing edge is moved downstream of the peak source. It is also possible to observe that the SPL variation decreases as the $L_{c}$ value decreases.)

(Added: The variation of $x_{T E}$ was useful to identify the effects of coherence when the plate is moved downstream of $X_{\max }$. However, the results for $x_{T E}=8 D$, and even $x_{T E}=6 D$, should be observed with care as this configuration probably violates the hypothesis of low influence of the plate over the jet flow field. In this sense, these results may not reproduce well the aspects of the real phenomenon.)

(Deleted: Analysis with line source)

(Deleted: Indeed, the isolated jet case is very sensitive to source variations and the radial profiles carry some complexity regarding the range of length seales, for example. It should be thus much simpler to fit a line source with radially integrated LES data. For doing that, a line souree was educed by considering radial compactness at low St. The derivation of the 
Free jet

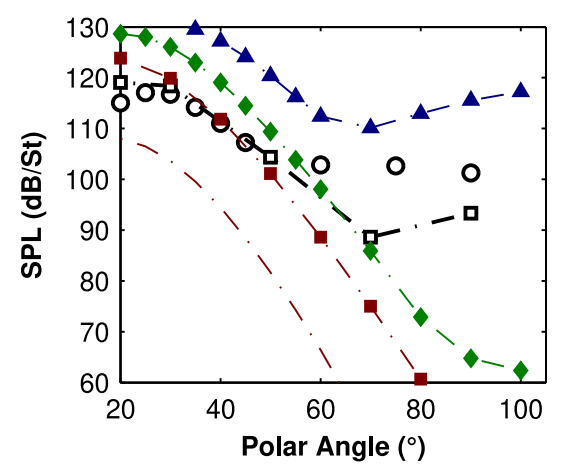

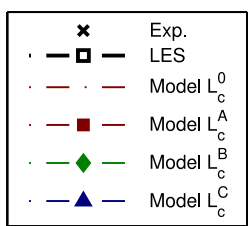

Installed Jet
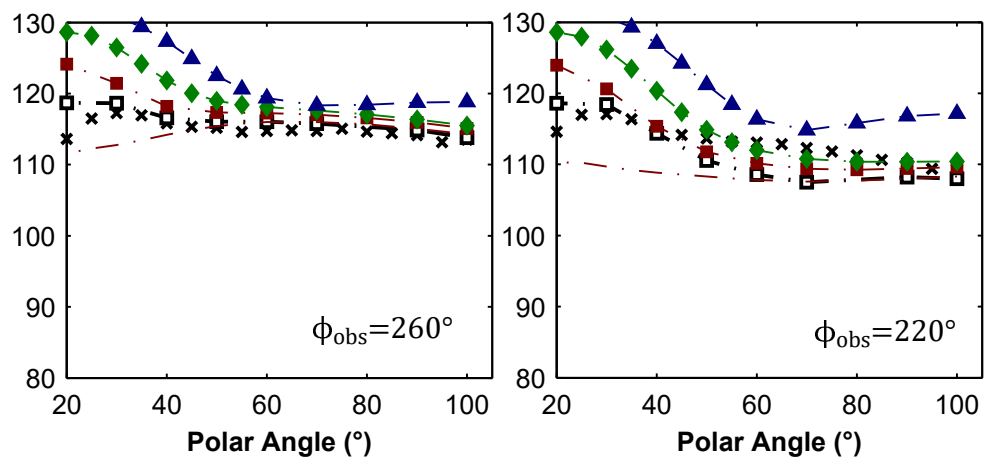

Scattering only
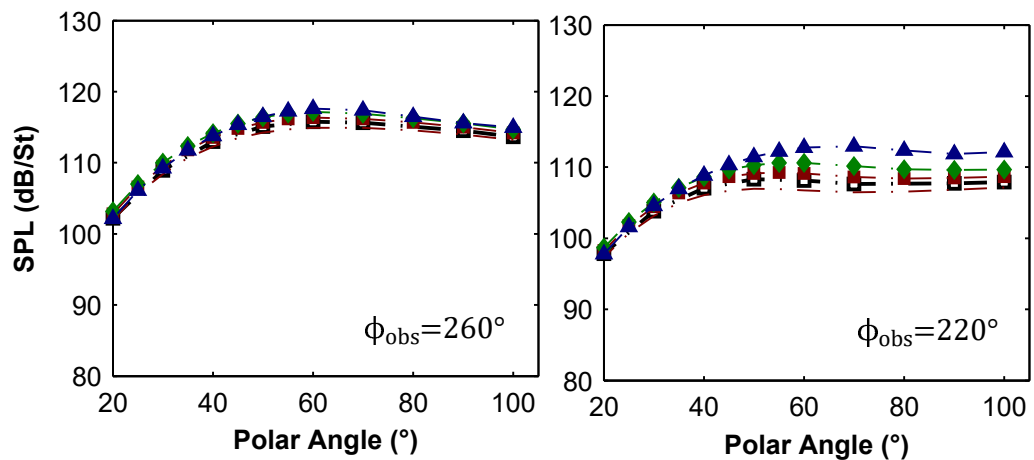

FIG. 8. (Color online) Model source results compared to the experiment and LES source for $S t=0.3$ with Free-field and tailored Green's function and varying $L_{c}$.

tine source is described in section $\mathrm{HE}$ and the model shown in Eq. ??. The source parameters are obtained by using the double radial integral of the LES source and are summarized in table??. This procedure is supposed to carry less uncertainties in the fitting process.)

(Deleted: Although the approximation sounds valid for the free jet, its application may be not accurate for the installed case, since the Green's function depends strongly on the distances between source and trailing-edge, which varies azimuthally. Assuming radial compactness in this case probably leads to larger errors for the installed case. To reduce the effects of these simplifications, a cylindrical-surface source, concentrated on the lipline 

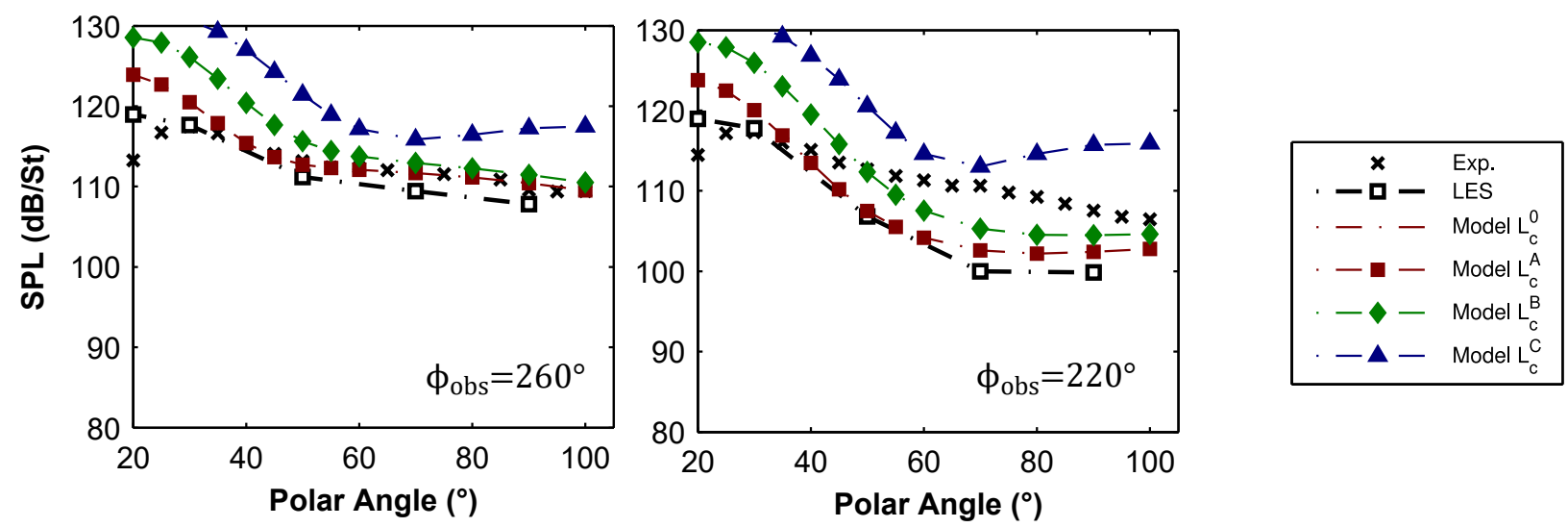

FIG. 9. (Color online) Model source results compared to the experiment and LES source for $S t=0.3$ with tailored Green's function for the plate at $r / D=1.5$ and varying $L_{c}$.
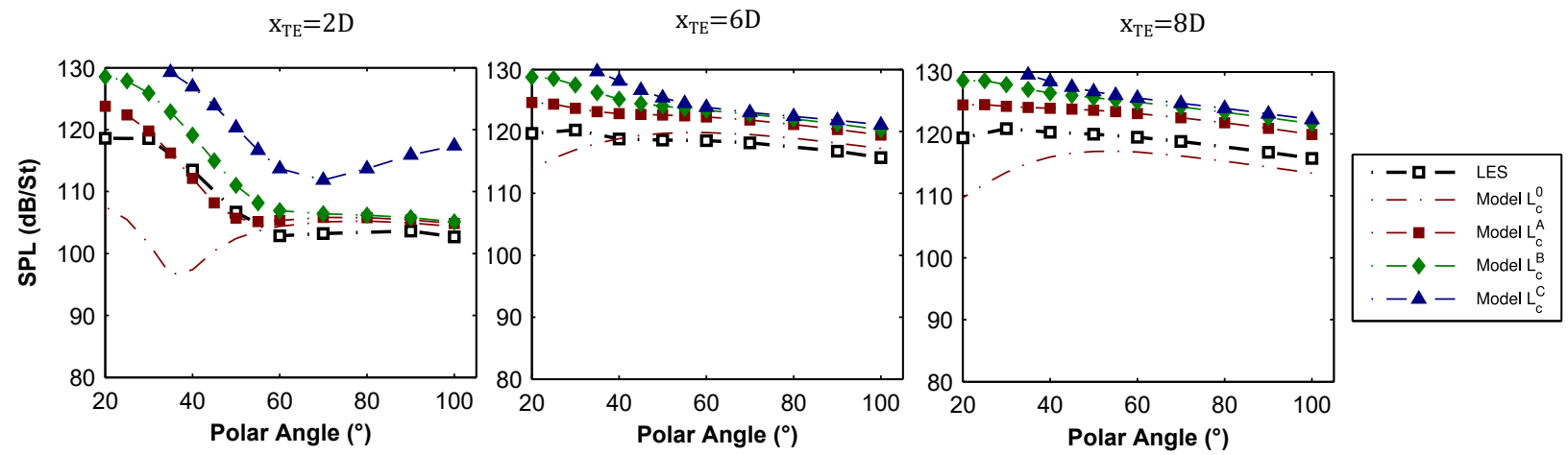

FIG. 10. (Color online) Model source results compared to the LES source for $S t=0.3$ with the plate at different axial positions and varying $L_{c}$. Observers at $\phi_{o b s}=260^{\circ}$.

is also used, as defined by Nogueira et al. ${ }^{12}$. The same parameters used for the line source are used in the cylindrical surface approach.)

(Deleted: Some of the parameters in Table ?? changed significantly from those in Table I. The axial position $X_{c}$ appears more upstream for all $S t$. The envelope length $L_{x}$ now decreases with St, a different trend from the two-dimensional fit. Higher values of $k_{h}$ were found for all $S t$.) 
(Deleted: Acoustic results are show in Fig. ??(a) for $S t=0.2$, comparing the line and eylindrieal sources. For the isolated jet, results showed a slightly better agreement with reference data in both the maximum amplitude and directivity when compared to the previously used source model. However, noise levels are still overpredicted by about $4 \mathrm{~dB}$. Regarding installed jet cases (Fig. ??(d)), the results for $S t=0.2$ are in very good agreement with the reference data, as well as the volumetric source. Minor differences can be noted between the line and cylindrical surface sources, even for the installed case with plate at $r / D=1$.

(Deleted: Free-jet results were also-slightly improved for $S t=0.3$ (Fig. ?? (b)). For the installed jet case (Fig. ?? (e)), the model results were about $4 \mathrm{~dB}$ higher than the reference data. Besides possible inaccuracies in the fitting process, it is important to emphasize that the use of the radially integrated data can lead to errors in the prediction of the jet-plate noise.)

(Deleted: Data for $S t=0.75$, depicted in Fig. ?? (c) reveals considerable differences between the cylindrical surface and the linear sources. The noise levels were overpredicted by about $45 \mathrm{~dB}$ by the line model and the cylindrical surface model could not correctly reproduce the directivity trends.)

(Deleted: Fig. ?? compares the radially integrated LES data with the fitted model sources with reference point at $X_{\max }$. We can now see that the model can reproduce the main eharacteristics of the LES source in terms of PSD, CSD and coherence for the chosen $S t$. Indeed it becomes much simpler to fit line information with simple Gaussian functions in eomparison to the two-dimensional cases presented on the previous sections. The diffieulties 
regarding the radial variation of the coherence lengthscale $L_{c}$, for instance, are overcome by eompacting the coherence information on a line.)

(Deleted: Fig. ?? shows that the asymmetric Gaussian function, with the obtained parameters, is suitable for modelling the PSD amplitudes. The amplitude and phases of the CSD were also well represented by the model at this position, as also shown in Fig. ??. It is important to note, however, that the shapes of both the CSD and coherence are not symmetric, especially for $S t=0.75$. In Fig. ??, for instance, the coherence shows a narrow peak, with rapid decay, followed by a much slower decay at the right side for $S t=0.75$. These features could not be well reproduced by the chosen Gaussian function.)

(Deleted: Even though the asymmetric shapes of both the CSD and coherence could not be completed represented by the used functions, it is clear the information was enough for a good and fast prediction of the trailing edge seattering noise. More complexity seems to be required for the isolated jet at higher $S t$, both regarding the radial information and the analysis of the coherence function. This is outside the scope of the present study.)

\section{CONCLUSION}

A study of jet-plate interaction noise was conducted using numerical data as input for wavepacket source models. A simplified source term was extracted from a numerical simulation database and the corresponding acoustic field was obtained for both the free-jet, by using the free-field Green's function, and for the installed jet by using a tailored function for a semi-infinite plate. After analysing the main characteristics of the simplified source term, 
the numerical data was used to adjust the parameters of simple source models. The noise prediction capabilities of such models were assessed for both isolated and installed jets.

The main features of the sound field for the isolated jet at low St and low polar angles could be obtained by considering only the linear part of the $T_{x x}(m=0)$ source term extracted from the LES. Moreover, this term was show to be the main contributor to the jet-plate scattered field, confirming the observations from 10. Significant sensitivity to the window function used on the calculation of the source CSD was observed. In general, results using rectangular window were not desirable and presented large sensitivity to the block size, in comparison to the Hanning windows. This suggested high sensitivity of the acoustic analogy to errors in the signal processing.

(Deleted: Two and one-dimensional) Source models were built, (Deleted: both) by minimising the squared error between the LES CSD and the proposed models. The sensitivity to the coherence length scales was analysed. By observing the CSD fields, the two-dimensional source approach could not correctly represent the characteristics of the source in every region. That may be the main cause for the found deviations on the free-jet noise results. Anyhow, the installed jet noise (Added: , for configuration in which trailing-edge scattering is relevant,) was seen to be much less sensitive to details in the modelled source.

(Deleted: The use of simpler source models, such as line source adjusted with radially integrated LES data, has proven to be more effective. This approach resulted in good agreement with experimental data, a consequence of involving less uncertainties in the fitting process. From the obtained results, we could observe that simplified models such as the ones 
tsed by 13 and 12 are able to correctly predict the noise levels for both installed and isolated jets, as long as they are parametrised with consistent source data.)

In summary, simple wavepacket models adjusted with flow field data, showed great capabilities to reproduce the noise level and directivity, specially for installed jets, with very low computational effort; kinematic wave-packet sources may be useful for parametric studies of jet and wing placement, highlighting configurations with lower sound radiation. The installed jet results were much less sensitive to fitting errors, specially to variations on the coherence parameters. In this way, modelling for installed jets becomes much simpler. (Added: It is also important to mention that the analyses were limited to installed configurations in which the jet flow is not significantly affected by the plate and so trailing-edge scattering would be the main installation effect.)

\section{ACKNOWLEDGMENTS}

The LES study was performed at Cascade Technologies, with support from NAVAIR SBIR project, under the supervision of Dr. John T. Spyropoulos. The main LES calculations were carried out on DoD HPC systems in ERDC DSRC. The authors also acknowledge the support

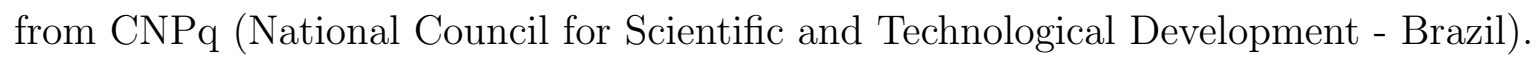

\section{REFERENCES}

${ }^{1}$ N. Curle, "The influence of solid boundaries upon aerodynamic sound," Proceedings of the Royal Society of London 231, 505-517 (1955). 
${ }^{2}$ M. J. Lighthill, "On sound generated aerodynamically. I. general theory," Proceedings of the Royal Society of London. Series A. Mathematical and Physical Sciences 211(1107), 564-587 (1952).

${ }^{3}$ J. E. Ffowcs Williams and L. H. Hall, "Aerodynamic sound generation by turbulent flow in the vicinity of a scattering half plane," Journal of Fluid Mechanics 40, 657-670 (1970). ${ }^{4}$ P. Jordan and T. Colonius, "Wave packets and turbulent jet noise," Annual Review of Fluid Mechanics 45(1), 173-195 (2013).

${ }^{5}$ E. Mollo-Christensen, "Jet noise and shear flow instability seen from an experimenter's viewpoint," Journal of Applied Mechanics 34(1), 1-7 (1967).

${ }^{6}$ S. C. Crow and F. H. Champagne, "Orderly structure in jet turbulence," Journal of Fluid Mechanics 48, 547-591 (1971) doi: 10.1017/S0022112071001745.

${ }^{7}$ C. E. Tinney and P. Jordan, "The near pressure field of coaxial subsonic jets," Journal of Fluid Mechanics 611, 175-204 (2008) doi: 10.1017/S0022112008001833.

${ }^{8}$ A. V. G. Cavalieri, P. Jordan, T. Colonius, and Y. Gervais, "Axisymmetric superdirectivity in subsonic jets," Journal of Fluid Mechanics 704, 388-420 (2012) doi: 10.1017/jfm. 2012.247.

${ }^{9}$ A. V. G. Cavalieri, D. Rodríguez, P. Jordan, T. Colonius, and Y. Gervais, "Wavepackets in the velocity field of turbulent jets," Journal of Fluid Mechanics 730, 559-592 (2013) doi: 10.1017/jfm.2013.346.

${ }^{10}$ A. V. Cavalieri, P. Jordan, W. R. Wolf, and Y. Gervais, "Scattering of wavepackets by a flat plate in the vicinity of a turbulent jet," Journal of Sound and Vibration 333(24), 
6516-6531 (2014).

${ }^{11}$ S. Piantanida, V. Jaunet, J. Huber, W. R. Wolf, P. Jordan, and A. V. G. Cavalieri, "Scattering of turbulent-jet wavepackets by a swept trailing edge," The Journal of the Acoustical Society of America 140(6), 4350-4359 (2016) doi: 10.1121/1.4971425.

${ }^{12}$ P. A. Nogueira, A. V. Cavalieri, and P. Jordan, "A model problem for sound radiation by an installed jet," Journal of Sound and Vibration 391, 95 - 115 (2017) doi: 10.1016/j. jsv.2016.12.015.

${ }^{13}$ A. V. G. Cavalieri and A. Agarwal, "Coherence decay and its impact on sound radiation by wavepackets," Journal of Fluid Mechanics 748, 399-415 (2014).

${ }^{14}$ A. V. Cavalieri, P. Jordan, A. Agarwal, and Y. Gervais, "Jittering wave-packet models for subsonic jet noise," Journal of Sound and Vibration 330, 4474 - 4492 (2011b).

${ }^{15}$ Y. B. Baqui, A. Agarwal, A. V. G. Cavalieri, and S. Sinayoko, "A coherence-matched linear source mechanism for subsonic jet noise," Journal of Fluid Mechanics 776, 235-267 (2015) doi: 10.1017/jfm.2015.322.

${ }^{16}$ D. Papamoschou, Prediction of Jet Noise Shielding, doi: 10.2514/6.2010-653.

${ }^{17}$ B. Lyu, A. P. Dowling, and I. Naqavi, "Prediction of installed jet noise," Journal of Fluid Mechanics 811, 234-268 (2017) doi: 10.1017/jfm.2016.747.

${ }^{18}$ B. Lyu and A. P. Dowling, "Modelling installed jet noise due to the scattering of jet instability waves by swept wings," Journal of Fluid Mechanics 870, 760-783 (2019) doi: $10.1017 / j \mathrm{fm} .2019 .268$. 
${ }^{19}$ G. A. Brès, P. Jordan, V. Jaunet, M. Le Rallic, A. V. G. Cavalieri, A. Towne, S. K. Lele, T. Colonius, and O. T. Schmidt, "Importance of the nozzle-exit boundary-layer state in subsonic turbulent jets," Journal of Fluid Mechanics 851, 83-124 (2018) doi: $10.1017 / j f m .2018 .476$.

${ }^{20} \mathrm{P}$. Welch, "The use of fast fourier transform for the estimation of power spectra: A method based on time averaging over short, modified periodograms," IEEE Transactions on Audio and Electroacoustics 15(2), 70-73 (1967) doi: 10.1109/TAU.1967.1161901.

${ }^{21}$ S. C. Crow, "Acoustic gain of a turbulent jet Phys. Soc. Meeting, Univ. Colorado, Boulder," paper IE, vol. 6. (1972).

${ }^{22}$ D. Crighton, "Basic principles of aerodynamic noise generation," Progress in Aerospace Sciences 16(1), 31 - 96 (1975) doi: 10.1016/0376-0421(75)90010-X.

${ }^{23}$ A. Michalke and H. Fuchs, "On turbulence and noise of an axisymmetric shear flow," J. Fluid Mech. 70, 179 (1975) doi: 10.1017/S0022112075001966.

${ }^{24}$ D. Juvé, M. Sunyach, and G. Comte-Bellot, "Filtered azimuthal correlations in the acoustic far field of a subsonic jet," AIAA Journal 17(1), 112-113 (1979) doi: 10.2514/3.61076.

${ }^{25}$ D. J. Bodony and S. K. Lele, "Low-frequency sound sources in high-speed turbulent jets," Journal of Fluid Mechanics 617, 231-253 (2008) doi: 10.1017/S0022112008004096.

${ }^{26}$ C. J. Mead and P. J. R. Strange, "Under-wing installation effects on jet noise at sideline," in 4th AIAA/CEAS Aeroacoustics Conference, AIAA 98-2207, American Institute of Aeronautics and Astronautics (1998). 
525

526

${ }^{27} \mathrm{~J}$. Lawrence, "Aeroacoustic interactions of installed subsonic round jets," PhD thesis, University of Southampton, Faculty of Engineering and the Environment, 2014.

${ }^{28}$ J. A. Nelder and R. Mead, "A simplex method for function minimization," The Computer Journal 7(4), 308 (1965) doi: 10.1093/comjnl/7.4.308.

TABLE I. Values of the resulting source parameters from the CSD fits. Non-dimensional values.

\begin{tabular}{cccccccccc}
\hline St & $A$ & $X_{c}$ & $L_{x}$ & $\alpha$ & $L_{r}$ & $k_{h}$ & $L_{c}$ & $\Delta$ \\
\hline 0.2 & $1.18 \times 10^{3}$ & 9.32 & 5.92 & 0 & 0.17 & 1.67 & 3.95 & 0.64 \\
\hline 0.3 & $0.31 \times 10^{3}$ & 3.26 & 9.45 & -5.06 & 0.16 & 2.6 & 3.00 & 0.63 \\
\hline 0.75 & $0.07 \times 10^{3}$ & 1.77 & 7.91 & -22.8 & 0.11 & 6.01 & 3.31 & 0.84 \\
\hline
\end{tabular}


Modelling of wavepacket scattering noise

\section{List of Changes}

Added: at the region dominated by trailing-edge scattering, on page 2, line 10 . 